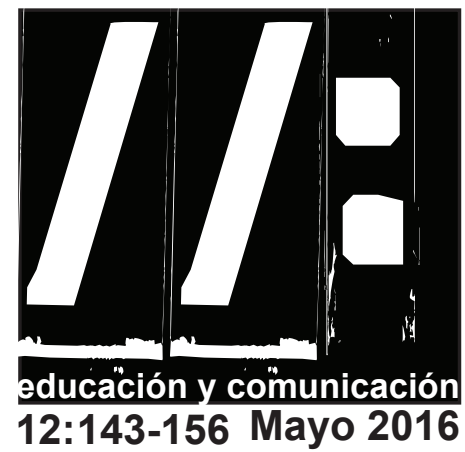

\title{
AGERCÁMDONOS A LA INTERCULTURALIDAD DESDE UNA VISIÓN INTEGRADORA DEL CURRÍCULO: LOS CUENTOS COMO TÓPICO ARTICULADOR
}

\section{Approaching interculture from an integrative sight of the curriculum: the stories like joining topic}

\author{
Valentina Rodríguez Sánchez \\ Manuel Fco. Romero Oliva \\ E.mail: manuelfrancisco.romero@uca.es \\ Universidad de Cádiz (España)
}

Resumen:

En el presente artículo se pretende crear un marco de reflexión y actuación sobre la importancia de la creatividad a través de la innovación y la integración del currículo para una educación intercultural, tomando como tópicos articuladores los cuentos populares y la interacción de las distintas lenguas del currículo desde la visión de un Proyecto Lingüístico de Centro (PLC) y el Currículum Integrado de las Lenguas (CIL).

Palabras claves: educación literaria, creatividad, competencia literaria e intercultural, PLC, AICLE, CIL

Abstract:

This article tries to create a frame of reflection and action about importance of creativity for innovation and integration of curriculum for an intercultural education, taking as joined topics folktales and interaction of the different languages of the curriculum from the vision of a Linguistic Project of Center (PLC) and the Integrated Curriculum of the Languages (CIL).

Key words: Literary Education, Creativity, Literary and Intercultural Competence, PLC, AICLE, CIL

Recibido: 31/12/2015 Revisado: 23/02/2016 Aceptado: 02/04/2016 Publicado: 01/5/2016 


\section{IIA Interculturalidad, educación y comunicación}

\section{Introducción}

E docente busca una educación que consiga emocionar e iluminar la oscuridad de lo desconocido. Como dice Pablo Neruda, es necesario confundirse, deshacer las cosas y recomponerlas para poder comprender con claridad lo que conllevan. De esta manera, es necesario buscar una educación que capacite a los más pequeños para hacer frente a este mundo, para que no solo lo conozcan, sino para que comprendan su funcionamiento y estructuras. Desde estos principios generales, esta propuesta pretende:

crear un marco de reflexión sobre la importancia de la creatividad a través de la innovación del currículo y la integración de diversas disciplinas; y, plantear una propuesta didáctica en donde se concrete la interacción de las distintas lenguas del currículo desde la visión de un Proyecto Lingüístico de Centro (PLC) y el Currículum Integrado de las Lenguas (CIL) para una educación intercultural.

En el trabajo se parte de una reflexión sobre las distintas teorías, enfoques y escritos para conformar la base de la propuesta que conlleve una mejora de la competencia comunicativa en todas las lenguas presentes en el aula. En este sentido, tomando como base el PLC, se pretende establecer una relación entre los contenidos de la lengua materna (L1) y la lengua extranjera (L2), desarrollando un enfoque de Aprendizaje Integrado de Contenidos y Lenguas (AICLE).

Hacia una educación plurilingüe e intercultural desde la didáctica de la lengua y la literatura
En la sociedad actual nos hallamos ante una ruptura virtual de fronteras que trae consigo la apertura a nuevas culturas, que no solo entran en la vida de los alumnos de forma física, mediante los procesos migratorios; sino también de forma virtual, haciendo que elementos de distintos lugares estén presentes en su vida cotidiana. Esta multiculturalidad presente en el medio conlleva la necesidad de desarrollar desde la escuela una conciencia intercultural que haga que los futuros ciudadanos valoren de forma real los intercambios culturales, asimilando la importancia del enriquecimiento mutuo entre las distintas sociedades. "En Didáctica de la Lengua la interculturalidad se ha convertido en poco tiempo en un objetivo de enseñanza paralelo a la propia competencia comunicativa" (Trujillo, 2005:24). La DLL contiene entre sus fines la consecución de una competencia comunicativa que permita llevar a cabo intercambios comunicativos en cualquier contexto, todos los aspectos sociales llevan intrínsecos un sistema simbólico que da existencia al mundo, este sistema simbólico está en estrecha relación con la cultura y las lenguas (Bruner, 1987 en Trujillo, 2005:25). De este modo, no se debe tratar la cultura como un conjunto de datos y costumbres, sino como una fuerza que ayuda a entender la realidad y actuar consecuentemente con ella (Trujillo, 2005:29). En esta línea Mendoza (2003:11, en Trujillo, 2005:32) asemeja la competencia intercultural a la competencia lingüística: "formar hablantes competentes y lectores eficaces, buenos redactores, individuos plenamente desarrollados y capaces de relacionarse con los demás". En el aula esto solo es posible a partir de la diversidad sociocultural, la variedad de experiencias comunicativas y un enfoque crítico de la enseñanza. 


\section{1: Acercandonos a la interculturalidad desde una visión integradora del currículo: los cuentos como tópico articulador}

Definiéndose la competencia plurilingüe e intercultural desde el Marco Común Europeo de Referencia para las Lenguas (2002:31) como "la capacidad de utilizar las lenguas para fines comunicativos y de participar en una relación intercultural en que una persona, como agente social, domina -con distinto grado- varias lenguas y posee experiencias de varias culturas", el docente, además de la propia diversidad del aula y otros factores propios del contexto, tiene la posibilidad de establecer una relación entre conocimiento, percepción y comprensión de la lengua materna y la estudiada para producir conciencia intercultural.

\section{Bases para una educación plurilingüe e intercultural desde el currículo.}

Las competencias -básicas o claves- están colmando, incluso saturando, los diferentes foros educativos sobre los procesos de enseñanza-aprendizaje del futuro. Una educación, basada en estas opiniones, pretende desarrollar en el alumno capacidades que le permitan resolver problemas cotidianos, actuales y futuros. Con este fin, se buscan nuevas formas de aprendizaje basadas en la interacción, el trabajo cooperativo, la interdisciplinariedad y el conocimiento global de la realidad, tomando como base un aprendizaje constructivo y significativo que permita una formación integral en el alumno (Prado, 2001:24). La integración de las competencias en el currículum educativo aúna en un mismo término tanto el ámbito escolar como el laboral, el personal, el interpersonal y el social; englobando saber, saber hacer, saber ser y saber convivir. La búsqueda de una formación integral y competencial encaja poco con los métodos tradicionales - clasificación de saberes en disciplinas y el "saber por saber" - se enfoca la enseñanza a un saber funcional, útil para la vida y la sociedad (Zabala y Arnau, 2014:9,10). En primer lugar, buscamos un aprendizaje significativo, basado en las relaciones sustanciales, no arbitrarias, entre los distintos saberes. De esta forma, se deja de lado una formación superficial que no cambia los esquemas mentales del alumno y que hace que los aprendizajes caigan en saco roto. De hecho, autores como Ausubel (1976:9) afirman que la mente humana no está diseñada para internalizar y almacenar asociaciones arbitrarias, necesita redes de conocimiento que permitan integrar los nuevos saberes. Para dotar los aprendizajes de significado se deben dar dos factores claves en el aula: una actitud positiva del alumno, que está dispuesto a relacionar, y no a memorizar sistemáticamente; así como tareas de aprendizaje significativas y relacionables con la estructura cognitiva del alumno (Ausubel, 1976: 2,3).

Además, la motivación es otro elemento clave para lograr una actitud positiva, "consiste en proporcionar a los alumnos un conjunto de motivos, incentivos o propósitos que produzcan el deseo de aprender" (Romero, 2009: 33), mediante el uso de estrategias diversas y tareas que lo sean por sí mismas, se debe hacer que el alumno vea sentido en su trabajo, un fin claro, comprensible y cercano en el tiempo, no entenderá que sea útil para su futuro. Un contexto de aprendizaje que implique al alumno y un ambiente libre de estrés lo alejará de memorización y las relaciones arbitrarias, para ello la concepción del "error" juega un papel clave. Si lo sancionamos no permitimos el desarrollo de un aprendizaje funcional y significativo, Ken Robinson (2006) establece que los niños arriesgan sin miedo y es la escuela la que mediante una educación negativa del error desarrolla el miedo a equivocarnos 


\section{II: Interculturalidad, educación y comunicación}

propio de los adultos, educamos en contra de la creatividad, no permitiendo desarrollar productos originales y creativos.

Así mismo, el nivel de las tareas y la dificultad que confortan es también un factor clave para la significatividad, las tareas demasiado difíciles podrían tomarse como imposibles y desistirían; demasiado fáciles aburrirían al alumno. Deben situarse en la Zona de Desarrollo Próximo (ZDP) del niño -entre el nivel real de desarrollo y el nivel de desarrollo potencial- (Vygotsky 1979:133 en Signored, 2009:321). Del mismo modo, una tarea no será significativa si el aprendizaje no se construye sobre las estructuras cognitivas que el alumno posee. Si los antecedentes de los aprendizajes escolares se dan por interiorizados, mientras han sido simplemente memorizados, no permiten incorporar, entender y fijar ideas nuevas, llevando a un fracaso de los procesos de enseñanza-aprendizaje, los procesos de acomodación o asimilación solo se dan si se conocen los esquemas sobre los que se quiere construir, estableciendo relaciones sustanciales y no arbitrarias (Ausubel, 1976:7,8). En palabras de J.P. Bronckart (2006:101), "todo saber nuevo no puede, pues, sino situarse en relación con ese saber adquirido para enriquecerlo, contestarlo o transformarlo".

Para lograr este aprendizaje funcional y significativo surgen en la actualidad nuevos enfoques y métodos de enseñanza. Entre ellos, decidimos destacar y profundizar en el enfoque globalizador y el trabajo por proyectos, que pretenden aunar los saberes escolares en torno a situaciones cercanas al alumno que le permitan relacionar saberes y situaciones que confluyen en el aula (escolar, cotidiano y propio del alumno). Se trata de abordar un conocimiento desde todas las vertientes, exprimiendo al máximo sus posibilidades, dejando que se relacionen y se nutran entre ellos para lograr un aprendizaje completo, significativo y funcional (Zabala y Arnau, 2014: 31-33).

La UNESCO define un proyecto como "una actividad práctica y significativa, de valor educativo, en relación con unas metas específicas de comprensión. Implica la investigación, resolución de problemas, y muchas veces, la utilización de objetos concretos. Esta actividad es planificada y llevada a cabo por los estudiantes y profesores en un entorno natural y verdadero" (Zabala y Arnau, 2014: 51). Un proyecto es un proceso de solución, elaboración o construcción con un objetivo claro, que lleva la consecución de unos contenidos competenciales. En el aula se traduce en un alumno con un papel activo, mientras que el docente pasa a ser un dinamizador, conductor y facilitador del proceso de aprendizaje, es decir, cambian los roles de la enseñanza tradicional en la que el alumno permanecía pasivo ante un profesor activo que poseía el conocimiento. El alumno actual debe aprender a manipular la información para convertirla en conocimiento, que ya no se proporciona por los materiales curriculares, este alumno busca, experimenta y comprende a través de diversas fuentes. Es así como la organización social del aula pasa a ser cambiante, los tiempos y el espacio se adaptan a las necesidades del proceso, superando los límites del aula y buscando una mayor relación con el contexto.

Respecto a la enseñanza de las lenguas, todo docente ha de orientar su acción docente desde "la didáctica para la formación de ciudadanos competentes y sujetos de su destino, que exige la capacidad de acceder a los conocimientos adquiridos, de apropiárselos, de contribuir a su transformación y, según la cual, es en este mismo dominio donde se forma -indirectamente- 


\section{1: Acercandonos a la interculturalidad desde una visión integradora del currículo: los cuentos como tópico articulador}

a la persona" (Bronckart, 2006: 106). En la formación de esta capacidad desempeña un papel protagonista la competencia comunicativa, concibiendo la lengua como reguladora del pensamiento y trasmisora de saberes, la verbalización de los conocimientos nos lleva al razonamiento de las ideas y la comprensión. La actividad lingüística es generadora de saber en la medida en que es la forma de materialización obligada de la actividad cognitiva (Bronckart, 2006:102).

Apoyando esta idea, Trujillo (2005) aporta a la lengua un papel central en la construcción de la persona mediante la cultura, la interacción es indispensable para el aprendizaje y solo podemos transmitir y recibir saberes a través de la materialización lingüística del pensamiento. Es así como surgen en la escuela diferentes iniciativas que aúnan lenguas y culturas, como son PLC (Proyecto Lingüístico de Centro), CIL (Currículo Integrado de Lenguas) o AICLE (Aprendizaje Integrado de Contenidos y Lenguas Extranjeras), que vienen a establecer una relación entre los contenidos de las diferentes áreas lingüísticas y no lingüísticas para superar las barreras entre estas, vinculándolo a los intereses del alumno y llevando un currículum global desde una educación plurilingüe que lleve a una mejora general de la competencia comunicativa y al desarrollo de una competencia pluricultural.

Finalmente, para la búsqueda de una mejora educativa, tan deseada por los docentes y necesaria para la sociedad, hemos de concretarla desde dos aspectos clave: la creatividad y la interculturalidad. Estas se establecen como herramientas clave para la formación de ciudadanos que sean competentes en la sociedad presente y futura. De este modo, el futuro se torna cada vez más impredecible por lo que se requieren ciudadanos con la capacidad de innovar y crear; la creatividad (Robinson, 2006), trata de formar personas capaces de interpretar problemas, buscando soluciones en varios y nuevos caminos. En cuanto a la interculturalidad, Trujillo (2005:35) establece que a lo largo de su vida cada sujeto desarrolla su identidad a partir de experiencias culturales y situaciones sociocomunicativas, la conciencia intercultural se vuelve indispensable para interaccionar con los conocimientos que llevan al éxito comunicativo y. por lo tanto, educativo y futuro.

El término creatividad está tomando cada vez más fuerza en el contexto educativo, pero su promoción es insuficiente. Hoy en día resulta difícil hallar una definición clara de creatividad, la RAE la define como “capacidad de creación”, entendiendo creación como algo nuevo que surge de la inventiva. Nos encontramos ante una definición que abarca una gran amplitud de conceptos y una palabra que evoca firmemente la libertad, por lo que la falta de claridad en el discurso educativo resulta más que evidente y la confusión entre los docentes está asegurada. Ken Robinson (2011) añade connotaciones a la definición: "proceso de tener ideas nuevas que sean valiosas", concibiendo la creatividad como una capacidad para solucionar problemas. Así mismo, resulta inevitable relacionar este concepto con novedad u originalidad, la diferencia reside en el valor de lo creativo, que además de nuevo y original resulta útil. Es, por ello, que la creatividad conlleva múltiples atributos que la originalidad no recoge, "envuelve tanto generar nuevas ideas como sintetizar una variedad de ideas ya concebidas dentro de una nueva forma de entendimiento" (Desailly, 2012). Trabajarla en el aula no debería resultar un trabajo difícil, los niños son creativos por naturaleza: arriesgan, aprenden a partir del tanteo, prueban, sin miedo 


\section{IIA Interculturalidad, educación y comunicación}

a equivocarse y buscan soluciones corriendo riesgos (Robinson, 2006). Para la elaboración de propuestas que fomenten la creatividad, la imaginación juega un papel principal y es la capacidad clave que todos los alumnos poseen y que se debe desarrollar potencialmente; pero, para crear, antes hay que imaginar. La clave está en plantear situaciones-problema que lleven a los alumnos a sentir la necesidad de preguntarse cómo actuar, situaciones que partan de un mismo punto y permitan elegir distintos caminos sin miedo a que no sea el correcto (Robinson, 2011). A su vez, todo este proceso resulta más efectivo dentro del trabajo en grupo, al desarrollarse habilidades sociales y emocionales a través de la interacción con los iguales y la necesidad de expresar sus propias ideas (Desailly, 2012). Establecer un ambiente que ayude a pensar y a buscar nuevas soluciones, que haga de nuestros alumnos personas capaces y con iniciativa, es nuestra tarea como docentes.

\section{Una propuesta para primer ciclo de educación primaria basada en los cuentos populares desde una visión intercultural}

En la tarea docente el maestro debe tener en cuenta que la escuela no es un elemento aislado, un pequeño mundo en el que los alumnos dejan en la puerta todo lo que la sociedad hace de ellos. Sociedad y escuela son dos realidades indivisibles, cada una de ellas debe aportar a la otra lo mejor de sí misma y exigirse a su vez que respondan a sus propias necesidades. La principal tarea de la escuela debe ser "ofrecer una enseñanza acorde con la realidad actual, que prepare un alumnado capaz de desenvolverse con autonomía en la misma” (Prado, 2001: 22).
Pensamos en un aula de segundo de primaria, edad en la que los alumnos desarrollan una capacidad de iniciativa que les lleva a incrementar el conocimiento, la habilidad para aprender y el uso de su propia imaginación. Buscan ser personas productivas, capaces de hacer cosas y resolver problemas, no sentirse capaces de esto conlleva una sensación de inferioridad, que resulta negativa para su desarrollo (Erikson, 1983 en Muñoz, 2010: 30). Desde una perspectiva constructivista, que centra las causas del desarrollo cognitivo en procesos internos, Piaget sitúa a los niños de primer ciclo en el paso del periodo preoperatorio a las operaciones concretas, que se caracteriza por la capacidad para realizar operaciones -transformaciones reversibles que afectan a los objetos-, "comunes a los individuos de un mismo nivel mental y que no solo intervienen en sus razonamientos privados sino también en sus intercambios cognitivos; reunir información, ponerla en acción o corresponder constituyen operaciones" (Piaget, 1997: 15).

La intervención del adulto, en este caso el profesor, para el desarrollo lingüístico es fundamental; el interaccionismo de Vygotsky (1979, en Signored, 2009: 321) concibe los contextos social, cultural y lingüísticos como elementos esenciales para el desarrollo. El niño aprende internalizando la cultura: se la apropia, la transforma y domina sus instrumentos a partir de la interacción. Esta intervención no puede ser cualquiera, debe situarse en la Zona de Desarrollo Próximo (ZDP) y estar acompañada por el apoyo del adulto, así es como Bruner en los años 70 parte de esta ZDP para desarrollar el concepto de andamiaje, que analiza de forma más exhaustiva los apoyos proporcionados por el adulto, basándose en los procesos de aprendizaje naturales que se dan en las relaciones madre-hijo. 


\section{1: Acercandonos a la interculturalidad desde una visión integradora del currículo: los cuentos como tópico articulador}

Del mismo modo, analiza la forma en la que los niños adquieren y usan la lengua materna, definiendo un sistema de apoyo -andamiaje- para la adquisición de cualquier lengua a partir del proceso de interacción entre el niño y el adulto. Se trata de diseñar un proceso de transmisión de habilidades en el que al principio los apoyos son mayores y progresivamente se van retirando hasta que se logra el aprendizaje. Este proceso es el mismo que se debe seguir para la adquisición de una lengua extranjera, creando así una similitud con la de la lengua materna, no basta con llenar la cabeza de información, hay que saber en qué momento debemos desarrollar cada habilidad y hacerlo de forma que esta se interiorice correctamente para que permita dar paso a nuevos aprendizajes.

Se desarrolla así una propuesta para trabajar un enfoque extensivo de las destrezas escritas en las lenguas del aula; pues se pretende, por un lado, fomentar el disfrute con la lectura y los talleres de escritura, y, por otro, desarrollar la autonomía y la creatividad en el alumno. Todo este proceso se encamina hacia la escritura creativa en el área de lengua extranjera (inglés), haciéndose necesario el aporte de destrezas y contenidos procedentes de otras áreas -desde esta perspectiva, se plantea la metodología AICLE y el enfoque interdisciplinar-.

Partiendo del nivel lingüístico del alumno, se interviene desde un enfoque intensivo en aspectos concretos de las lenguas que permiten crear el andamiaje necesario para abordar los contenidos desde un planteamiento extensivo, donde el alumno activa los conocimientos adquiridos para la realización de un proyecto. Este último permite una visión global de los conocimientos y supone ir un paso más allá en los procesos de enseñanza-aprendizaje de las destrezas escritas: alumnos lectores, que disfruten leyendo; y alumnos escritores, que usen la creatividad para escribir.

Enmarcada dentro de la metodología AICLE, se muestra la lengua como vehículo de comunicación y herramienta para el aprendizaje, buscando desarrollar en el aula métodos interactivos, significativos y constructivistas (Galera, 2005: 45, en Romero, 2009: 192). En este caso, las lenguas confluyentes en el aula son trabajadas desde un mismo punto, intentando crear puentes y paralelismos entre sus elementos lingüísticos y culturales. Este proceso se ajusta a las características del proceso natural de aprendizaje, centrándose en la adquisición de la lengua, ajustándose al momento y al proceso evolutivo del alumno, sus capacidades y necesidades; ofreciendo actividades, recursos y dinámicas de aula que supongan una iniciación placentera en la L2 y motiven al alumnado a ser partícipe de su propio proceso de aprendizaje (Fernández, J., 1997). La propuesta se compone de actividades para el aprendizaje -estudio consciente, formal y explícito- y para la adquisición -codificación o decodificación de mensajes- de la L2 (Krashen, 1982 en Romero, 2009:35), que se dividen siguiendo las siguientes fases que respetan el proceso natural de adquisición de la lengua, yendo de las destrezas orales a la expresión escrita.

El desarrollo de este proyecto se sustenta en dos pilares fundamentales entendidos como un todo: la lectu-

\begin{tabular}{|c|ccc|}
\hline $1^{2}$ FASE & $2^{2}$ FASE & $3^{2}$ FASE & $4^{2}$ FASE \\
\hline $\begin{array}{c}\text { COMPRENSIÓN } \\
\text { ORAL }\end{array}$ & $\begin{array}{c}\text { PRODUCCIÓN } \\
\text { ORAL }\end{array}$ & $\begin{array}{c}\text { COMPRENSIÓN } \\
\text { ESCRITA }\end{array}$ & $\begin{array}{c}\text { PRODUCCIÓN } \\
\text { ESCRTTA }\end{array}$ \\
\hline
\end{tabular}

Gráfico 1: Fases para la adquisición de una lengua extranjera, por Romero (2009:35) 


\section{II: Interculturalidad, educación y comunicación}

ra y la escritura. Leer es una actividad que precisa de la activación de una triple vertiente conceptual: experiencias previas, elementos cognitivos (conocimientos) y actitudes. La lectura es una excusa para desarrollar el resto de destrezas comunicativas, poniendo a los alumnos en situación de creación y producción. Para ello, se intercalan continuamente las diferentes destrezas, pasando de las orales a las escritas, de las comprensivas a las expresivas, haciendo especial hincapié en el dialogo para la construcción de sentido y el desarrollo de saberes. La decodificación da paso a la comprensión y la interpretación, llevando este proceso más allá de las habilidades trabajadas desde una metodología tradicional (Romero, 2009), conectándose a su vez, de forma indirecta, con otras materias y contenidos del currículum, llevando a cabo un trabajo interdisciplinar que ayude al desarrollo de enfoques globales. Es así como se pretende desarrollar en el aula un proyecto para la creación de un producto a partir de la activación de los conocimientos adquiridos, que dote al aprendizaje de sentido y haga al alumno partícipe del proceso.

Desarrollar un proyecto en el aula permite al alumno intervenir en el proceso de forma activa, a través de tareas individualizadas que permitan un desarrollo competencial y un aprendizaje flexible, adaptable a ritmos y necesidades. Se pretende crear una propuesta integradora, que permita a todos intervenir en ella según sus capacidades e intereses, con el trabajo cooperativo no se deja de lado a alumnos con necesidades educativas especiales, evitando hacer adaptaciones. Los objetivos se presentan abiertos y flexibles, por lo que la consecución de estos vendrán definidos por el nivel de desarrollo competencial alcanzado por cada alumno, en función de sus propias características.
Para acentuar la flexibilidad se presenta una propuesta con una temporalización orientativa, los tiempos vendrán definidos por los propios alumnos, que los adaptarán a la situación contextual.

\section{Estrategias didácticas}

Toda metodología debe estar basada en unos fundamentos teóricos y llegar al aula a través de unas estrategias que permitan ponerlos en práctica. En este caso se destacará el uso de los cuentos populares como elemento motivacional e intercultural, así como la disposición de aula y el trabajo en grupo para un aprendizaje significativo.

\section{a) Cuentos populares}

Los cuentos populares son elementos del folclore infantil con gran valor para los primeros cursos de primaria, como afirma María Teresa Rodríguez (1991:2) "los materiales procedentes de la tradición oral propician un aprendizaje significativo de la lengua extranjera, además de tener otras características educativas, lingüísticas, lúdicas y afectivas de valor didáctico". En la misma línea, Magaly Villarrael (2002) defiende que los cuentos en el aprendizaje de la L2 crean una actitud positiva en el alumno, haciendo del aula un contexto atractivo que facilita el aprendizaje. A su vez, son una herramienta útil para desarrollar la imaginación y la creatividad, así como para el desarrollo integral de la persona, "las historias en general contribuyen a educar en valores, a la comprensión de problemas y conflictos, lo que hace que niños y niñas elaboren juicios y adopten comportamientos adecuados" (Villarrael, 2000:1).

Por su esquema narrativo sencillo, así como por su léxico, estructuras y temáticas recurrentes, los fairy 


\section{1: Acercandonos a la interculturalidad desde una visión integradora del currículo: los cuentos como tópico articulador}

tales se convierten en piezas de lectura y audiciones excelentes para los primeros cursos (Rodríguez, 1991:2,3), al leerlos en el aula con apoyos visuales dotamos al alumno de un excelente Input Comprehensible (Krashen en Cancelas, 1997:41). Entre sus principales características cabe destacar la acción única, los personajes antitéticos y el argumento sencillo y repetitivo que se encuentra en todos ellos y que confiere seguridad al lector al eliminar la incertidumbre y el estrés de no poder predecir los hechos. Del mismo modo, "la creación de libros caseros (home-madebooks) servirá para que los niños aprendan a apreciar y valorar los libros, se familiaricen con ellos y para estimular su creatividad" (Cancelas, 1997:40), estos suponen una técnica de animación a la lectura en un proceso que aúna todas las destrezas comunicativas realizado a través de actividades lúdicas, motivantes y de carácter interdisciplinar.

Los cuentos populares proceden del contexto cultural de la lengua, son propios de unas o se versionan según la zona o el país. Esto les confiere un gran valor cultural que no debe pasarse por alto en la enseñanza de lenguas. Desde esta propuesta se pretende contribuir no solo al conocimiento multicultural de los alumnos, sino de forma más precisa, al desarrollo de una competencia intercultural, paralela a la competencia lingüística que logre llevar el plurilingüismo más allá, hacia la interculturalidad (Trujillo,2005). Desde el desarrollo de esta competencia se pretende hacer que paulatinamente la integración en un centro multicultural se convierta en una realidad, más allá de intenciones y teorías.

\section{b) Organización de aula y trabajo cooperativo}

Excepto en los momentos de trabajo en grupo, los alumnos se disponen en forma de herradura -"U"-, lo cual favorece la interacción, la conversación y el intercambio de ideas. Para las explicaciones y los momentos centrados en el docente los alumnos están sentados mientras nos desplazamos por el aula. Para trabajos individuales o grupales se permite la movilidad por el aula para ayudarse entre ellos y solventar dudas, con esta movilidad y libertad en el trabajo se liberan tensiones y estrés, haciéndolo más agradable. Se tiene en cuenta que estamos ante alumnos pequeños, con una necesidad de movimiento muy alta, no se puede esperar que permanezcan, toda la mañana, sentados, quietos y callados; es necesario atender a sus necesidades como niños.
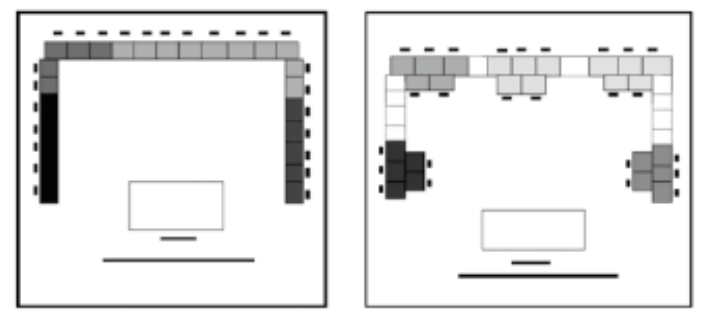

Gráfico 2: Organización espacial del aula para una metodología interactiva, según Romero (2009: 193).

Por otra parte destacamos el papel del trabajo cooperativo y el aprendizaje entre iguales para un aprendizaje significativo, "el conocimiento es una construcción social, donde el sujeto es constructor del conocimiento $\mathrm{y}$, el sujeto, a su vez, se construye conjuntamente con el objeto de conocimiento" (Rojas, 2001:5). En esta construcción el dialogo y la interacción son elementos clave, atendiendo al siguiente cuadro es más sencillo comprender por qué: 


\section{II: Interculturalidad, educación y comunicación}

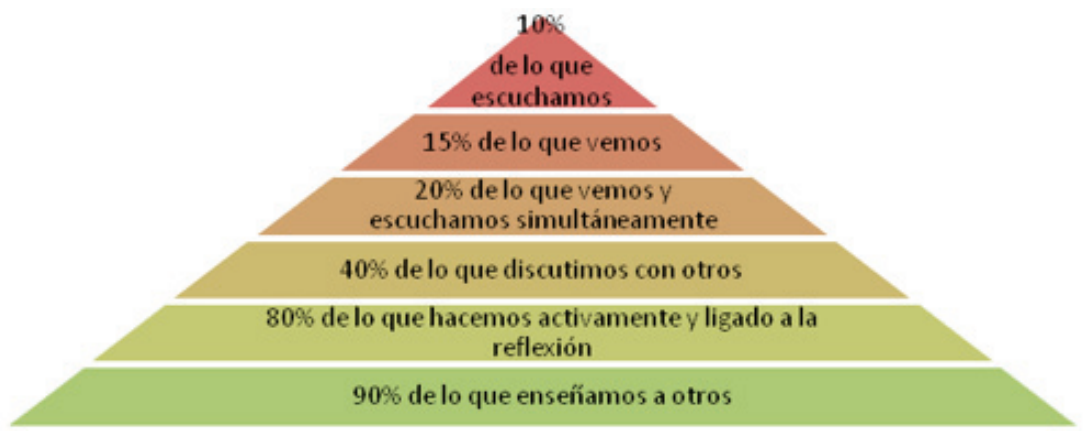

Gráfico 3: Estudio sobre la retención del aprendizaje elaborado por Philips en 1991, por A. Zabala y L. Arnau (2014): 3.

Este gráfico nos muestra cómo con métodos en los que el alumno se mantiene pasivo se interioriza menos de la cuarta parte del total de la información, mientras que estos porcentajes se elevan notablemente con un papel activo del alumno. Una metodología que tiene como pilar el aprendizaje cooperativo engloba los tres niveles de la base de la pirámide, por lo que los resultados en el aprendizaje se ven claramente favorecidos. Si el alumno participa activamente entra, a través de la discusión, en un conflicto cognitivo en el que se cuestiona el propio aprendizaje, alcanzando mayores cotas de aprendizaje.

\section{Descripción de actividades: de la L1 a la L2.}

La propuesta comienza con una primera toma de contacto con el tema; para ello, se realiza en pequeño grupo una serie de actividades de clasificación y búsqueda de recursos procedentes del folclore infantil en la lengua materna. Es necesario aclarar que en un primer momento las actividades se realizan en la L1 pasando luego al trabajo en la lengua extranjera y estableciendo así relación entre ambas. Esta primera sesión termina con la escritura grupal por parte de los alumnos del cuento que servirá como eje durante todas las sesiones: Caperucita Roja.

Con esta propuesta se produce el tránsito de la lectura a la escritura a través del dialogo y el trabajo en las distintas destrezas comunicativas. Para ello, en la segunda sesión, se proporciona a los grupos de alumnos las producciones que sus compañeros escribieron en la sesión anterior. Se realiza una lectura grupal apoyada en el dialogo y una serie de micro-actividades de decodificación que permita familiarizar a los alumnos con el léxico y las estructuras que se trabajarán posteriormente en la lengua extranjera. Pasaremos seguidamente a una comprensión más profunda del texto mediante la estructuración, secuenciación y síntesis de las ideas principales y secundarias. Por último, para reforzar esta comprensión, los alumnos ilustran y reescriben el cuento estableciendo una escena para cada idea de la secuenciación realizada durante las actividades de comprensión. En el último momento del proceso, se pide a los alumnos que reflexionen sobre 


\section{1: Acercandonos a la interculturalidad desde una visión integradora del currículo: los cuentos como tópico articulador}

su trabajo, comprobando los resultados y realizando las modificaciones oportunas.

En la siguiente actividad se lleva a cabo un proceso lecto-escritor, con la misma estructura que el realizado en la actividad anterior, pero en lengua extranjera. Comenzamos con la lectura del cuento Caperucita Roja -Little Red Hiding Hoof-, al ser en la L2, debemos ser conscientes de que es un proceso más complejo que en lengua materna, y más a estas edades. Es, por ello, que se lleva a cabo en tres momentos distintos:

1. Se comienza contando el cuento con ayuda de flashcards, el profesor habla y los alumnos escuchan. Esto también sirve de motivación, para captar la atención e introducir a los alumnos en la actividad. Este apoyo visual que proporcionan las flashcards se refuerza con la entonación y gesticulación.

2. En un segundo momento, se pasa a la lectura del cuento en la pizarra digital o a formato papel del texto. Este se acompaña de imágenes que ayudan a la construcción del significado del vocabulario y la propia comprensión del texto. El profesor realiza una lectura expresiva mientras los alumnos, a su vez, siguen la lectura en voz baja, interiorizando el texto.

3. Por último, son los alumnos los que realizan la lectura. El profesor lee cada frase pidiendo luego a los alumnos que la lean en voz alta de forma individual. No se corrige de forma exhaustiva, ya que se busca el disfrute con la lectura.

Una vez realizada la lectura, se trabajan tanto vocabulario como estructuras, pasando de comprender a inferir para desarrollar en los alumnos las herramientas para la producción:

- Primero los alumnos ven las imágenes en flas- hcards, escuchan y repiten las palabras. Posteriormente se les presenta la palabra escrita y deben relacionar están con la idea que representan, por último buscan nuevas palabras y las escriben. Del mismo modo con las estructuras pasan de escucharlas a crearlas ellos mismos para formar frases. - Para reforzar la comprensión del cuento, el vocabulario y las estructuras se lleva a cabo una actividad de relación de las ideas principales del cuento, en la L2, con la ilustraciones que realizaron anteriormente, así podemos comprobar si han sido capaces de comprender la historia y relacionar dicha comprensión con el vocabulario y las estructuras.

- Estamos realizando en el aula un proyecto cuya idea está encaminada a la elaboración de un álbum ilustrado por parte de los alumnos en pequeños grupos. Para ello, se les proporciona una plantilla de escritura usando la estructura del cuento de Little Red Hiding Hoof y eliminando palabras, permitiendo adaptar la actividad a distintos niveles sin que cambie su desarrollo, objetivos o contenidos. Del mismo modo, se darán flashcards con el vocabulario usado y los alumnos completarán las oraciones incompletas con nuevas palabras, creando así una nueva historia que debe de tener sentido, -además realizarán ilustraciones que acompañen a la historia para ayudar a su comprensión-.

- Una vez finalizada la actividad, se les pide que reflexionen sobre su trabajo. Para ello vuelven a leer las secuencias con las imágenes y piensan si hay concordancia ilustración-texto, de no ser así será el momento de reelaborar y mejorar.

- Por último, desplazaremos a los alumnos a la 


\section{IIA Interculturalidad, educación y comunicación}

biblioteca del centro para presentar y leer a sus compañeros las historias que han creado. Entre todos seleccionan las dos que más les han gustado para compartirlas e introducirlas en el proyecto lingüístico del centro, que podemos denominar "Talking-art".

\section{Conclusiones}

En nuestro trabajo, hemos pretendido aportar líneas para una educación que capacite a los más pequeños para hacer frente a este mundo, que no solo lo conozcan, sino que comprendan su funcionamiento y estructuras. En este sentido, es necesario dejar a lado la saturación de contenidos abstractos e individualismo; para construir una escuela que proporcione una visión real de su entorno desde los planteamientos cooperativos de la educación. De esta forma, estaremos abriendo a los alumnos un mundo de posibilidades para que lleguen a ser ciudadanos creadores de una sociedad mejor y capaces de ser felices en ella porque comprenderán e interpretarán la realidad en la que se desarrollan.

Es, pues, en una época en la que todo es efímero, que la educación se ve expuesta a construir sobre unos cimientos débiles e inestables, apostamos por una enseñanza global y un trabajo interdisciplinar que propicie un desarrollo integral del alumno para convertirlo en una persona competente en la vida real, interesada por el mundo circundante e interrelacionado con lo más lejano; es, por ello, que la escuela debe ofrecer espacios compartidos para una educación plurilingüe e intercultural.

\section{Referencias}

Ausubel, D. P., Novak, J. H. H. (1976). Significado y aprendizaje significativo. Psicología educativa: un punto de vista cognoscitivo. México: Trillas.

Bronckart, J. P. (2006). La transposición didáctica en las intervenciones formativas. Desarrollo de la educación y educación para el desarrollo integral. Contribuciones desde la Pedagogía del Texto. Medellín (Colombia): Universidad de Medellín, IDEA.

Cancelas, L. (1997). "Home-Made Books". Literatura Infantil y Juvenil, 126.

Desailly, J. (2012). Creativity in the primary classroom. Londres: SAGE Publications.

Fernandez, José (1997). "La literatura infantil en la iniciación a la lengua extranjera". Actas I Jornada de Didáctica de la Lengua y la Literatura Infantil y Juvenil. Servicio de Publicaciones de la Universidad de Cádiz.

Ministerio de Educación, Cultura y Deporte (2002). Marco Común Europeo de Referencia para las Lenguas: aprendizaje, enseñanza, evaluación. Madrid: Secretaría General Técnica del MECD-Subdirección General de Información y Publicaciones y Grupo ANAYA.

Muñoz García, A. (2010). Psicología del desarrollo en la etapa de educación primaria. Granada: Pirámide.

Piaget, J., Inhelder, B. (1997). Psicología del niño. Madrid: Ediciones Morata.

Prado, J. (2001). "La competencia comunicativa en el entorno tecnológico: un desafío para la enseñanza". Comunicar: Revista Científica de Comunicación y Educación, 17.

Robinson, K. (2006). TED talks, 19:24; Do schools kill creativity? Recuperado el 16 de junio de 2015 


\section{1: Acercandonos a la interculturalidad desde una visión integradora}

\section{Divulgatio}

de http://www.ted.com/talks/lang/eng/ken_robinson says_schools_kill_creativity.html

Robinson, K., Punset, E. (2011). Los secretos de la creatividad. En Redes, nº8. Recuperado el 16 de junio de 2015 de https://www.youtube.com/ watch? $\mathrm{v}=\mathrm{TOHaSdZfwP} 4$

Rodríguez, M.T. (1991). "Valores didácticos de la literatura popular y del folclore infantil inglés". Signos teoría y práctica de la educación, 3 .

Rojas, L. (2001). "Tratamiento de los contenidos lingüísticos y gramaticales en los libros de texto de la serie: hacia el siglo XXI". Educación: Revista de la Universidad de Costa Rica, vol. 25 no 2.

Romero, M.F. (2009). "Dominio de la expresión a través de mecanismos de motivación y auto-evaluación”. Entre Lenguas, Vol. 14.

Romero, M.F. (2009). “Acceso a las competencias básicas educativas desde la lecto-escritura". Tabanque Revista Pedagógica, 22.
Signored Dorcasberro, A. (2009). “Cognición, pensamiento y lenguaje: Perspectivas teóricas desde la psicolingüística, la adquisición del lenguaje y psicología cognitiva”. Estudios de Lingüística Aplicada, 50.

Trujillo, F. (2005). "En torno a la interculturalidad: reflexiones sobre la cultura y comunicación para la didáctica de la lengua”. Portal Linguarum, 4.

Vigotsky, L. S. (2007). Pensamiento y habla. Buenos Aires: Colihue.

Villarrael, M. (2000). "Los cuentos en el aula de primaria". Recuperado el 22 de agosto de 2015 de http:// www.rinconmaestro.es/profes/documentos/profes08. pdf

Zabala, A., Arnau, L. (2014). Métodos para la enseñanza de las competencias. Barcelona: GRAÓ 